\title{
Energy Dependence of Quasiparticle Relaxation in a Disordered Fermi Liquid
}

\author{
T. Schmidt, ${ }^{1, *}$ P. König, ${ }^{1}$ E. McCann, ${ }^{2}$ Vladimir I. Fal'ko, ${ }^{2}$ and R. J. Haug ${ }^{1}$ \\ ${ }^{1}$ Institut für Festkörperphysik, Universität Hannover, Appelstrasse 2, 30167 Hannover, Germany \\ ${ }^{2}$ Physics Department, Lancaster University, LA1 4YB Lancaster, United Kingdom
}

(Received 15 February 2000)

\begin{abstract}
A spectroscopic method is applied to measure the inelastic quasiparticle relaxation rate in a disordered Fermi liquid. The quasiparticle relaxation rate $\gamma$ is deduced from the magnitude of fluctuations in the local density of states, which are probed using resonant tunneling through a localized impurity state. We study its dependence on the excitation energy $E$ measured from the Fermi level. In a disordered metal (heavily doped GaAs) we find that $\gamma \propto E^{3 / 2}$ within the experimentally accessible energy interval, in agreement with the Altshuler-Aronov theory for electron-electron interactions in diffusive conductors.
\end{abstract}

DOI: $10.1103 /$ PhysRevLett.86.276

The quasiparticle description of electrons represents a common approach to understanding kinetic and thermodynamic phenomena in metals. It relies on a certain stability of quasiparticle excitations in a many-body system, which requires that the quasiparticle excitation energy, $E$, determined with respect to the Fermi level, $E_{F}$, exceeds the broadening of such a single-particle state, $\hbar \gamma(E)$, due to electron-electron interactions.

In ideally pure metals, the relaxation rate, $\gamma$, of ballistic quasiparticles at low energies is kept low by a diminishing of the phase space available for inelastic electron-electron collision processes [1]. A typical process is shown in Fig. 1. The phase space argument results in $\gamma \sim E^{2}$ dependence, which has been confirmed by electron emission spectroscopy measurements which probe electron states at energies $E \gg \hbar / \tau$, where $\tau$ is the elastic mean-free path time due to residual impurities. Quasiparticle decay at excitation energies $E<\hbar / \tau$ is accelerated by the presence of disorder (which makes the electron states chaotically random at length scales longer than the elastic mean-free path, $\left.l=\tau v_{F}\right)$. The theory of interaction effects in diffusive media [2] predicts that, at zero temperature, the decay of quasiparticle excitations with $E \ll \hbar / \tau$ in a disordered metal or heavily doped semiconductor is slow enough to guarantee the existence of properly defined quasiparticles. In particular, in bulk three-dimensional $(d=3)$ and two-dimensional $(d=2)$ conductors, the quasiparticle relaxation rate is expected to obey a power-law dependence,

$$
\gamma(E)=a E^{d / 2} E_{F}^{(1-d / 2)}\left(\lambda_{F} / l\right)^{d / 2} .
$$

Despite the fundamental interest and clear theoretical predictions [2], there are very few direct experimental measurements of the quasiparticle decay time in disordered metals at small excitation energies $E<\hbar / \tau$. Information about the inelastic decay of nonequilibrium quasiparticles in dirty metals is often extracted from energy relaxation rates in the electron thermalization process [3], $\gamma_{E}$. Otherwise, one studies the temperature dependence of a dephasing rate, $\gamma_{\varphi}(T)$, of coherent carriers (determined using weak localization or universal conductance fluctua-
PACS numbers: 73.23.-b, 72.20.My, 85.30.Mn

tion analysis), which can be treated as a measure of the efficiency of the interactions of an electron with energy $E \leq T$ with equilibrium fluctuations of charges produced by other electrons thermally distributed near the Fermi level in a diffusive conductor [4]. At high temperatures, both phase and energy relaxation experiments show a certain agreement with theoretical estimations [5-8]. However, recently reported data [3,9] on two dynamical parameters mentioned above, $\gamma_{E}(E)$ and $\gamma_{\varphi}(T)$, in $\mathrm{Au}$ wires and films, and also in semiconductor heterostructures [8], have indicated a certain disagreement between theoretically predicted and experimentally observed values of these two quantities, which has refueled both theoretical and experimental interest in the problem of quasiparticle lifetimes in a disordered metal [10].

In this paper, we report the results of a direct measurement of the energy dependence of the inelastic decay rate $\gamma(E)$ of a quasiparticle state in a disordered conductor. This study employs the method of resonant tunneling spectroscopy using a discrete localized state in a double-barrier structure, which was applied earlier by Geim et al. [11] to study 2D electrons in a heterostucture and by Sivan et al. [12] to investigate the discrete spectra of quantum pillars. It was shown previously [13-17] that, by measuring the current-voltage $(\mathrm{b} I-V)$ characteristics and by deriving the

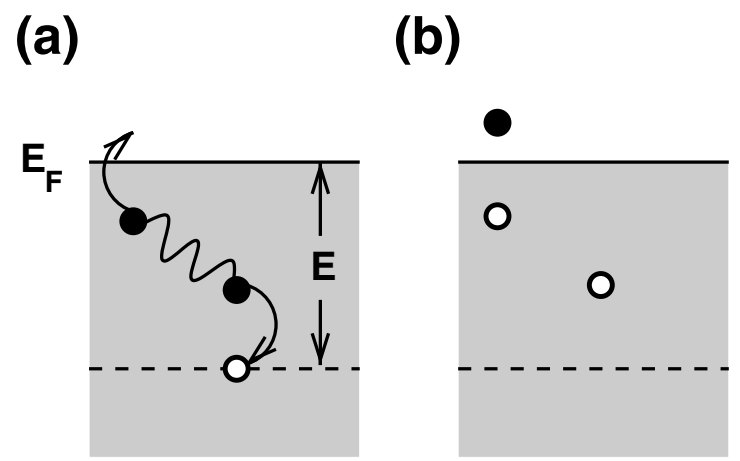

FIG. 1. A typical inelastic relaxation process: a quasihole $(\bigcirc)$ at energy $E$ below the Fermi level $E_{F}$ (a) decays into a quasiparticle () and two quasiholes (b). 
differential conductance in a system where the current passes through a single resonant impurity state in the barrier, one can study features of the single-particle spectrum of a disordered metal (playing the role of an emitter). In a bulk material with a continuous spectrum, individual chaotic quantum states formed by the interference of elastically scattered electrons produce an effect known as fluctuations of the local density of states (LDOS) [18]. It consists of a random and coordinate-specific energy dependence of the local density of states in a diffusive metal, $\nu(E)$, with a correlation energy limited by inelastic broadening of quasiparticle states, $\hbar \gamma$. We observe a random pattern in $\nu(E)$ by sweeping a single resonant impurity level against the electron spectrum in the emitter within a finite range of excitation energies for a quasihole (an empty state below $E_{F}$ in the emitter) left behind by the tunneled electron. When the energetic width of an impurity level used in this process, $\Gamma$, is smaller than the inelastic broadening of single-particle levels in the emitting electrode, $\hbar \gamma$, one can extract the latter characteristic from the analysis of the amplitude of the LDOS fluctuations pattern and its autocorrelation parameters. This has enabled us to measure directly the energy dependence of the inelastic relaxation rate, $\gamma(E)$, in bulk degenerate heavily doped GaAs at low temperatures, which we find to agree with the Altshuler-Aronov theory [2,19] predicting $\gamma(E)=a\left(E^{3 / 2} / \sqrt{E_{F}}\right)\left(\lambda_{F} / l\right)^{3 / 2}$ for a 3D system. [Note that, under the condition of $\hbar \gamma(E) \ll E$ provided in Eq. (1) for $\lambda_{F} \ll l$, the inelastic decay rate $\gamma$ of a nonequilibrium quasiparticle coincides with its decoherence rate.]

As mentioned above, the LDOS can be measured via resonant tunneling through an impurity in a strongly asymmetric double-barrier heterostructure. Our microstructure consists of a $10 \mathrm{~nm}$ wide GaAs quantum well and 5 and $8 \mathrm{~nm}$ wide $\mathrm{Al}_{0.3} \mathrm{Ga}_{0.7}$ As barriers sandwiched between doped GaAs contact layers with a donor concentration of $3.3 \times 10^{17} \mathrm{~cm}^{-3}$, as sketched in Fig. 2(a). From this material we fabricated a $2 \mu \mathrm{m}$ diameter mesa, as depicted in the scanning electron micrograph of Fig. 2(b). The mesa contains a small number of residual impurities in the nominally undoped quantum well. The energetically lowest impurity state $S$ in the well is used as a spectrometer for the LDOS imaging in the metallic emitter adjacent to the thicker barrier, as illustrated in Fig. 2(c). At zero bias, $S$ lies above the Fermi level in the emitter and is not available for resonant transport, resulting in $I=0$ and $G=0$. This measurement has been performed at temperature $T=20 \mathrm{mK}$. Upon applying a finite bias voltage $V$, the energetic position of a spectrometer $\mathrm{S}$ is shifted down to the energy $E=\alpha e\left(V-V_{S}\right)$ below the Fermi level in the emitter, where the prefactor $\alpha=0.50$ accounts for the fact that only part of the voltage drops between the emitter and the spectrometer [20]. When $S$ crosses $E_{F}$ from above (at $V_{S}=9.8 \mathrm{mV}$, the current acquires a finite value (plateaus) limited by the left, less (a)

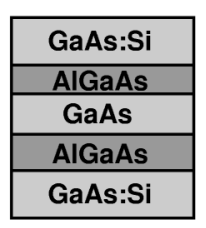

(b)

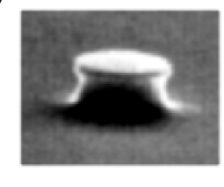

(c)

(d)
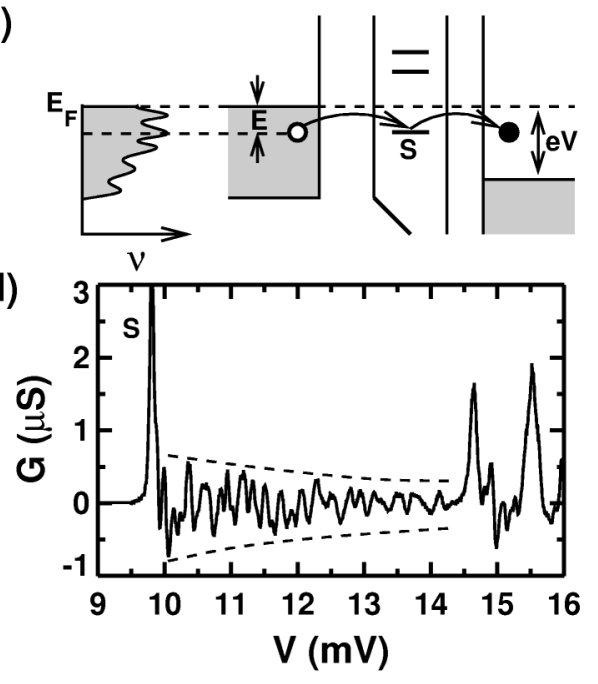

FIG. 2. (a) Schematic of a strongly asymmetric double-barrier heterostructure containing residual impurities in the quantum well. (b) Scanning electron micrograph of the fabricated mesa. The disk on top of the mesa is a metallization which serves as Ohmic contact. (c) Conduction-band profile of the device under operation. (d) Differential tunneling conductance $G=$ $d I / d V$ numerically obtained from $I(V)$ data recorded at the base temperature of $T=20 \mathrm{mK}$ of our dilution refrigerator.

transparent, barrier and proportional to the LDOS of occupied states in the emitter at the spectrometer position, $I \propto \nu[13,15]$. At higher bias voltages $\left(V_{H 1}=14.6 \mathrm{mV}\right.$ and $V_{H 2}=15.5 \mathrm{mV}$ ), other impurities (or, maybe, other excited states from the same impurity) become involved in the current formation, which produces the next prominent current steps in the $I-V$ characteristics. Consequently, the differential conductance $G=d I / d V \propto d \nu(E) / d E$ of the device plotted in Fig. 2(d) exhibits several pronounced peaks, the "main" one at $V_{S}=9.8 \mathrm{mV}$ followed by two at 14.6 and $15.5 \mathrm{mV}$, each characterizing the energetic width and transparency of a resonant impurity state, whereas in between, at $V_{S} \leq V \leq V_{H 1}$, the differential conductance displays the derivative of the LDOS with respect to energy.

The energy dependence of the LDOS is the result of the energy-dependent quantum interference pattern for quasiparticles in the emitter at the coordinate of a spectrometer [see Fig. 2(c)]. This pattern is random and tends to reflect an individual portrait of a disordered potential in a metal surrounding the spectrometer. In the sample under investigation, such a pattern can be analyzed within the energy interval of about $0 \leq E \leq 2 \mathrm{meV}$ below the Fermi level, since the voltage and energy scales are related via $E=\alpha e\left(V-V_{S}\right), \alpha \approx 0.5$. The correlation 
energy, $E_{c}=\Gamma+\hbar \gamma$, of the fine-structure in the differential conductance pattern is determined by either the energetic spectrometer width, $\Gamma$, or by the inelastic broadening of states in the emitter, $\hbar \gamma$, whichever is larger. According to the theory [15], one can relate $E_{c}$ to the amplitude (r.m.s. value) of the fluctuation pattern of $G(V) \propto$ $d \nu(E) / d E$. Note that, although oscillations at larger energy scales are also present in each realization of $\nu(E)$, their contribution to $G(V)$ is suppressed, due to the differentiation. For a given sample, the spectrometer width, $\Gamma$, can be extracted from the width of the main resonance peak. For the peak at $V_{S}=9.8 \mathrm{mV}$ in Fig. 2(d), we find $\Gamma \approx e \alpha \times 72 \mu \mathrm{V} \approx 36 \mu \mathrm{eV}$. For a broad spectrometer, with $\Gamma \gg \hbar \gamma(E)$ at any excitation energy [13], both the amplitude and the correlation voltage (energy) of fluctuations would be the same over the entire range of $V_{S} \leq V \leq V_{H 1}$. For a narrow spectrometer, such as studied in this paper, inelastic broadening of states in the bulk exceeds the spectrometer width upon increasing the excitation energy of a quasihole left in the emitter. This results in a decrease of fluctuations upon increasing voltage, as indicated by the dashed lines in Fig. 2(d).

Quantitative information about the quasiparticle decay rate, $\gamma(E)$, is obtained from statistical analysis of the complete fluctuation pattern $G(V, B)=d I / d V$ studied as a function of a magnetic field, $B$ (applied parallel to the current flow). Figure 3 shows a grayscale image of the differential conductance measured as a function of bias voltage and magnetic field (in the region of low magnetic fields, where Landau quantization is hindered by disorder). Sharp black lines in Fig. 3 correspond to the spectrometer crossing the emitter Fermi level. The decrease of the ampli-

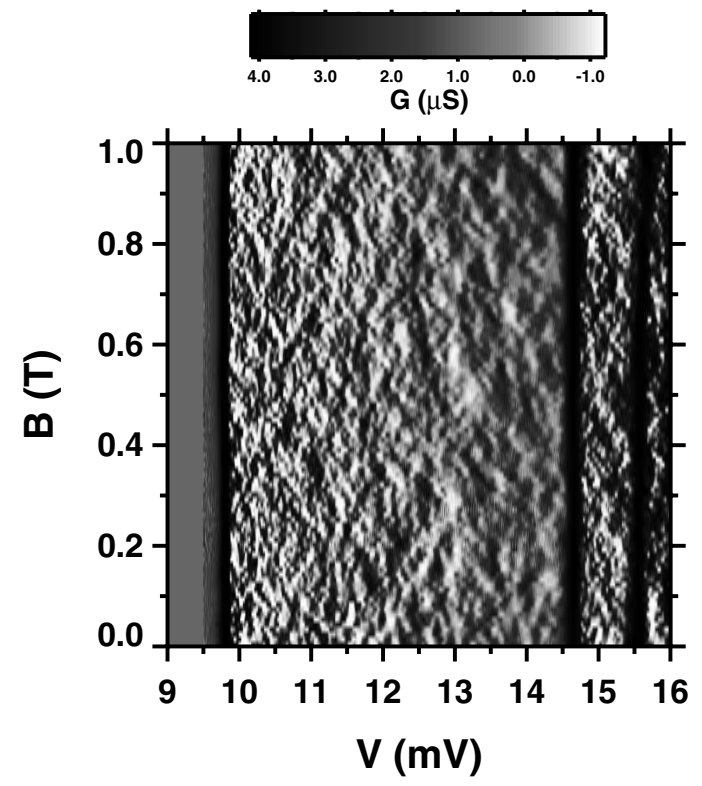

FIG. 3. Map of the differential conductance as a function of bias voltage (step $7 \mu \mathrm{V}$ ) and magnetic field $B \| I$ (step $10 \mathrm{mT}$ ) for $T=20 \mathrm{mK}$, which indicates that the magnitude of the fluctuations decreases with increasing bias. tude of observed LDOS fluctuations and the increase of the correlation voltage of the fluctuation pattern, as a function of the quasihole excitation energy, are apparent from the change in the contrast of this image.

As a quantitative measure of the fluctuation amplitude, we calculate the variance $\operatorname{var}_{B} G=\left\langle\delta G^{2}(B)\right\rangle_{B}$ using $\delta G(B)=G(B)-\langle G\rangle_{B}$, where $\langle\cdots\rangle_{B}$ indicates averaging over a magnetic field in the range of $0 \leq B \leq 1.0 \mathrm{~T}$. Figure 4(a) shows that $\operatorname{var}_{B} G$ drops by more than 1 order of magnitude within the experimentally accessible voltage range. In our limit of classical magnetic fields, the fluctuation amplitude is related to the relaxation rate [15] according to

$$
\left.\operatorname{var}_{B} G\right|_{V}=G_{N}^{2} \times[1+\hbar \gamma(E) / \Gamma]^{-3 / 2} .
$$

Here, $G_{N}$ is a prefactor which we determine as $G_{N}^{2}=$ $\left.\operatorname{var}_{B} G\right|_{V_{S}}$ from Fig. 4(a) by assuming that, at $V=V_{S}$ (corresponding to $E=0), \hbar \gamma \ll \Gamma$ and $E=\alpha e\left(V-V_{S}\right)$ is
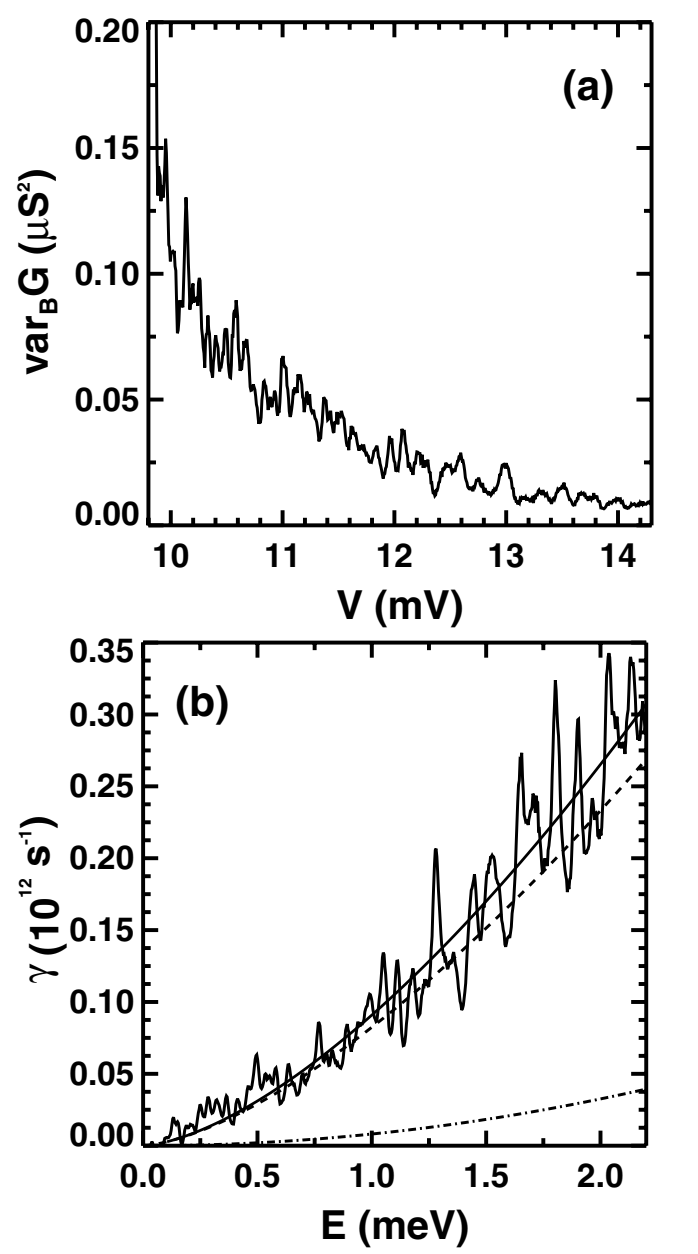

FIG. 4. (a) Variance of the differential conductance vs bias voltage. (b) Quasiparticle relaxation rate plotted as a function of excitation energy measured from the Fermi level. The experimental data are compared with a fit according to theory for disordered Fermi liquids, $\gamma=b E^{2}+A E^{3 / 2}$ (solid line). The individual terms $b E^{2}$ (dash-dotted line) and $A E^{3 / 2}$ (dashed line) are shown as well. 
the excitation energy of a quasihole (we remind the reader that $\alpha \approx 0.5$ in this experiment).

Figure 4(b) shows the obtained energy dependence of the quasiparticle decay rate. It drops strongly upon decreasing the excitation energy, and, in contrast to some experiments measuring dephasing rates [9], we do not observe a saturation of $\gamma(E)$ at low energies. In Fig. 4(b) we also compare the experimentally determined quasiparticle relaxation rate with the values calculated using the Altshuler-Aronov theory of electron-electron interaction in disordered conductors $[2,19]$. It is worth mentioning that the above-presented determination of the quasiparticle decay rate based upon the LDOS pattern analysis enables us to study quasiparticles with pretty small excitation energies, $E<\hbar / \tau$, and to detect the features of their inelastic decay specific to strongly disordered systems. This makes it different from the analysis of the same quantity on the basis of measurements of Landau level broadening [21], which requires distinct Landau quantization and a strong magnetic field (or absence of impurities).

Electron-electron scattering with a large momentum transfer between ballistic quasiparticles results in a rate which is determined by the phase volume of available final states, thus leading to $\gamma \propto E^{2}$ [1]. In disordered Fermi liquids, where transport is diffusive, small momentum transfers play an important role, such that an additional $E^{3 / 2}$ energy dependence of $\gamma$ appears, as described in Eq. (1). The $E^{3 / 2}$ dependence dominates at small energies, $E<\hbar / \tau$, while the $E^{2}$ dependence is specific to large energies, $E \gg \hbar / \tau$. After estimating the elastic scattering time of $\tau=0.14 \mathrm{ps}$ from the magnetic-field dependence of the LDOS fluctuations [22] (and also from the nominal doping level of the emitter contact), we find that the latter crossover would occur at $E \sim 5 \mathrm{meV}$, which is beyond the energy range accessible in the reported experiment. For a quantitative comparison, we fit the relaxation rate as $\gamma=b \times E^{2}+A \times E^{3 / 2}$ (dashed lines in Fig. 4(b) show separately the $b \times E^{2}$ and $A \times E^{3 / 2}$ parts determined in this fit). We have also fit the data to $\gamma(E)=A \times E^{x}$ dependence, treating exponent $x$ as a free parameter, which yields $x=1.54 \approx 3 / 2$ and $A=8 \times 10^{10} \mathrm{meV}^{-3 / 2} \mathrm{~s}^{-1}$. In the theory [19], the prefactor $A$ in the $E^{3 / 2}$ dependence is $A=(105 \sqrt{3 \hbar}) /\left(16 \pi \tau^{3 / 2} E_{F}^{2}\right)$. After estimating $E_{F}=26 \mathrm{meV}$ from the electron density in the emitter, we evaluate $A \approx 1 \times 10^{11} \mathrm{meV}^{-3 / 2} \mathrm{~s}^{-1}$ which compares well with the experimental result.

In conclusion, we presented a measurement of the inelastic quasiparticle relaxation rate in a disordered Fermi liquid. This quantity was obtained from the analysis of the magnitude of disorder-induced fluctuations in the local density of states probed using the method of resonant tunneling through an impurity state. Quantitative compari- son with the standard theory [2] shows that, within the energy range available for such an analysis, the experimentally determined values of the inelastic relaxation rate can be attributed to the electron-electron interaction relaxation mechanism in diffusive conductors.

We thank A. Förster and H. Lüth for growing the doublebarrier heterostructure. We acknowledge financial support from BMBF, DFG, EPSRC, NATO, and TMR.

*Present address: Goldman Sachs \& Co. oHG, Messeturm, Friedrich-Ebert-Anlage 49, D-60308 Frankfurt am Main, Germany.

[1] D. Pines and P. Nozieres, Theory of Quantum Liquids (Benjamin, New York, 1966).

[2] B. L. Altshuler and A. G. Aronov, in Electron-Electron Interactions in Disordered Systems, edited by A. L. Efros and M. Pollak (North-Holland, Amsterdam, 1985).

[3] H. Pothier et al., Phys. Rev. Lett. 79, 3490 (1997).

[4] Y. Imry, Introduction to Mesoscopic Physics (Oxford University Press, Oxford, 1997).

[5] G. Bergmann, Phys. Rep. 107, 1 (1984).

[6] S. Washburn and R. A. Webb, Rep. Prog. Phys. 55, 1311 (1992).

[7] R. M. Clarke et al., Phys. Rev. B 52, 2656 (1995).

[8] A. G. Huibers et al., Phys. Rev. Lett. 81, 200 (1998); A. G. Huibers et al., Phys. Rev. Lett. 83, 5090 (1999).

[9] P. Mohanty, E. M. Q. Jariwala, and R. A. Webb, Phys. Rev. Lett. 78, 3366 (1997).

[10] B.L. Altshuler, M.E. Gershenson, and I.L. Aleiner, Physica (Amsterdam) 3E, 58 (1998); I. L. Aleiner, B.L. Altshuler, and M.E. Gershenson, Waves Random Media 9, 201 (1999); Yu. B. Khavin, M.E. Gershenson, and A. L. Bogdanov, Phys. Rev. Lett. 81, 1066 (1998).

[11] A. K. Geim et al., Phys. Rev. Lett. 72, 2061 (1994).

[12] U. Sivan et al., Europhys. Lett. 25, 605 (1994).

[13] T. Schmidt et al., Europhys. Lett. 36, 61 (1996).

[14] J. W. Sleight et al., Phys. Rev. B 53, 15727 (1996).

[15] V. I. Fal'ko, Phys. Rev. B 56, 1049 (1997).

[16] T. Schmidt et al., Phys. Rev. Lett. 78, 1540 (1997).

[17] J. P. Holder et al., Phys. Rev. Lett. 84, 1563 (2000).

[18] I. V. Lerner, Phys. Lett. 133, 253 (1988); I. V. Lerner and M. E. Raikh, Phys. Rev. B 45, 14036 (1992).

[19] U. Sivan, Y. Imry, and A. G. Aronov, Europhys. Lett. 28, 115 (1994).

[20] The value $\alpha=0.5$ has been deduced from temperaturedependent $I(V)$ data following B. Su, V. J. Goldman, and J.E. Cunningham [Phys. Rev. B 46, 7644 (1992)], and M. R. Deshpande et al., [Phys. Rev. Lett. 76, 1328 (1996)].

[21] P. C. Main et al., Phys. Rev. Lett. 84, 729 (2000).

[22] The elastic scattering time $\tau$ has been obtained from the measured magnetic-field dependence of the variance at high magnetic fields using $\operatorname{var} G /\left.\operatorname{var} G\right|_{B=0}=1+\left(\omega_{c} \tau\right)^{2}$, where $\omega_{c}=e B / m^{*}$ is the cyclotron frequency [15]. 